This document is the Accepted Manuscript version of a Published Work that appeared in final form in Journal of Chemical Theory and Computation 11, 1745 (2015), copyright (c) American Chemical Society after peer review and technical editing by the publisher. To access the final edited and published work see

http://pubs.acs.org/doi/abs/10.1021/ct501160k 


\title{
Hydrated electrons in water clusters: inside or outside, cavity or non-cavity?
}

\author{
László Turi ${ }^{1}$ \\ Eötvös Loránd University, Department of Physical Chemistry, Budapest 112, P. O. Box \\ 32, H-1518, Hungary
}

We compare the applicability of three electron-water molecule pseudopotential models in modeling hydrated electron physical properties. The analysis is based on a) simple quantum mechanical model calculations, and b) one-electron mixed quantum-classical molecular dynamics simulations of an excess electron in size selected water cluster anions. The quantum mechanical calculations illustrate that the recently suggested Larsen-Glover-Schwartz (LGS) model predicts a too attractive potential in the vicinity of the oxygen. As a result, the LGS ground state eigenvalue and the asymptotic behavior of the model wave function are inaccurate. The TuriBorgis (TB) potential used for comparative purposes reproduces these properties satisfactorily. Mixed quantum-classical molecular dynamics simulations on negatively charged water clusters provide an ideal test case for further testing the potentials. In addition to the LGS and TB models, we also investigated a modified form of the LGS model (m-LGS) that were introduced to correct the huge overbinding of the electron in bulk LGS simulations. While the LGS and m-LGS

${ }^{1}$ E-mail: turi@chem.elte.hu, fax: (36)-1-372-2592. 
models predict non-cavity hydrated electron structure in clusters at room temperature, the TB potential prefers the traditionally accepted cavity structure. As another major difference, the electron exclusively localizes in the interior of the clusters in LGS based simulations, while two possible isomers (interior vs. surface state isomers) emerge from TB calculations. The computed associated physical properties are also analyzed and compared to available experimental data. We found that the LGS and m-LGS potentials provide results that are inconsistent with the size dependence of the experimental data. In particular, LGS simulations fail to reproduce the trends of the radius of the excess electron and the position of the absorption spectra with cluster size. The simulated TB tendencies are qualitatively correct. In conclusion, we observe that the cavity preferring pseudopotential model results physical properties in significantly better agreement with experimental data than the models predicting non-cavity structure for the hydrated electron. 


\section{Introduction}

The physical chemistry community has witnessed a wave of scientific activity in the last few years concerning an interesting, exotic species, the hydrated electron. ${ }^{1}$ Although the hydrated electron, an excess electron in a water bath, is a highly reactive species, ${ }^{2}$ it is thought to be an important intermediate in various fields. ${ }^{3}$ The scientific relevance of the hydrated electron stems from this curious chemical reactivity, but also from its significance as a simple, nevertheless instructive model system. Beyond the scientific research, even basic textbooks can benefit from the pictorial representation of the hydrated electron as a truly quantum particle interacting with its solvent environment (see Figure 3 later). The renewed (or better to say, continuous) research interest in the species is fueled by experiments employing the most sophisticated laser techniques to explore hydrated electron properties in the femtosecond $d^{4,5,6,7,8,9,10,11}$ or even in the attosecond time regime, ${ }^{12}$ and by theoretical attempts to provide a consistent molecular level picture underlying the experimental measurements. ${ }^{13,14,15}$

Molecular dynamics (MD) simulations have proved to be very efficient at rationalizing experimental observations and have converged during the years to a rather satisfying picture of the hydrated electron, as have been reviewed recently. ${ }^{16}$ Molecular dynamics on hydrated electron has been predominantly applied in the one-electron approximation, treating the electron quantum mechanically, while modeling the solvent environment by classical force fields ${ }^{17,18,19,20}$ in a mixed quantum-classical MD (QCMD) framework. ${ }^{21,22,23}$ Many electron ab initio molecular dynamics (AIMD) simulations, that are becoming gradually the choice of the method, are aimed at the more precise characterization of the system. ${ }^{13,24,25,26,27,28,29}$ Problems of AIMD, however, still persist in terms of the selection of the electronic structure calculation method, the simulated 
system size, and the sampling efficiency. These limitations of AIMD equally contribute to the fact that QCMD methods are still widely applied, even though these latter methods can be viewed realistically as semi-quantitative. ${ }^{16}$ We note here that the classical approximation of the solvent bath represents the main limitation of the applicability of the QCMD techniques. Nevertheless, it has been repeatedly pointed out that the most critical ingredient of the QCMD methods determining the physical viability of the simulated properties is the one-electron pseudopotential model that describes the interaction between the classical water bath and the quantum mechanical electron. Most of the conceptually important problems on the hydrated electron topic were recognized within the one-electron QCMD context, and are all closely related to the applied electron-water molecule pseudopotential. One such prominent problem concerns the structure of the hydrated electron. Although there are reasonable models in the literature that differ in the identification of the hydrated species (i.e. an excess electron, ${ }^{30}$ a solvent-anion complex, ${ }^{31} \mathrm{a}$ hydronium cation - hydoxyl anion complex,${ }^{32}$ a hydronium radical ${ }^{33,34}$ ), the dominantly accepted, more-or-less consensus model - the cavity model - proposes that a single electron is localized in a spatial void (cavity) in the water bath surrounded by oriented water molecules. ${ }^{30,35,36}$ This model was inferred and examined very early, ${ }^{30}$ and then later supported by one-electron path-integral Monte $\mathrm{Carlo}^{37}$, path-integral molecular dynamics ${ }^{36,38}$ and QCMD simulations. ${ }^{18}$ Without completeness we mention that the early pseudopotentials of Schnitker and Rossky, ${ }^{39}$ Barnett et al., ${ }^{40}$ Borgis and Staib, ${ }^{23}$ as well as the more recent models of Turi and Borgis, ${ }^{41}$ and Jacobson and Herbert ${ }^{42}$ all lead to cavity structure in QCMD simulations. The recently developed pseudopotential by Larsen, Glover and Schwartz (LGS) resulted in a completely different type of electron distribution, a non-cavity type structure for the bulk hydrated electron. ${ }^{43}$ In the noncavity model ("inverse-plum"44) the electron is delocalized over several water molecules in a 
region of enhanced water density. The electron may also localize in cavities but these voids are short living, collapse and then re-form very quickly. Inconsistencies in the Larsen potential were soon pointed out based on two independent approaches. A quantum mechanical analysis of a simple model and a subsequent quantum simulation showed that the LGS model is too attractive around the oxygen atom, and a simple modification of the potential leads to cavity structure in QCMD simulations. ${ }^{45}$ A quantum chemistry based analysis pointed out significant deviations between the predictions of the LGS model and the results of ab inito benchmark calculations for small static water cluster anions. ${ }^{46}$ A comparative study evaluating the applicability of the LGS model, the Turi-Borgis (TB), and the Jacobson-Herbert $(\mathrm{JH})$ model in simulations for the bulk hydrated electron was also published demonstrating that the basic physical properties associated with the cavity model are in more acceptable agreement with experiments than those computed with the LGS non-cavity model. ${ }^{47}$ Nevertheless, the application of the LGS model for the calculation of the resonance Raman spectrum of the hydrated electron produced a consistent picture with experiment, while cavity models seem to fail in this respect. ${ }^{44}$

Another challenging structural issue arises in connection with the presence of interfaces in finite size hydrated electron systems. It is generally accepted that the experimentally observed distinct excess electron vertical detachment energy (VDE) patterns with cluster size reflect the existence of distinct water cluster anion structural motifs (isomers). However, there is still no consensus whether the excess electron is localized in the interior or on the surface of the clusters. ${ }^{10,15,48,49,50,51,52,53,54,55}$ Clusters with different localization modes (i.e. interior state clusters vs. surface state clusters) are expected to exhibit different physical properties, and different trends of these properties with cluster size. Typical examples are the radius of the excess electron, the optical absorption spectra and the lifetime of the electronic excited states..$^{10,15,48,49,53,55}$ Of the 
cavity models, the JH and TB models reproduce the experimental VDE tendencies in a semiquantitative manner, but their assignments do not necessarily agree with the tentative experimental assignments. ${ }^{15,53,55}$ This unsettled problem provides an ideal testing ground where one can compare and contrast the predictions of the non-cavity LGS model with experiments and with the results of the cavity preferring potentials. Furthermore, cluster calculations can be related to bulk hydrated electron data by extrapolating cluster results to the bulk. This cluster extrapolation approach can be helpful to evaluate and remove artifacts that may be associated with bulk simulations. Potential problems include the application of finite simulation box with periodic boundary conditions, and the neglect or non-complete inclusion of long-range interactions. Such complex comparison and analysis are the main goal of the present paper. We will evaluate structural, energetic and spectroscopic properties of various size water cluster anions computed in QCMD simulations using two pseudopotential models, one predicting noncavity structure (LGS model), and another with cavity structure (TB model) in bulk hydrated electron simulations. The reason for the choice of the latter potential is that both the LGS and the TB potentials employ the same non-polarizable water-water classical potential (simple point charge (SPC) model with flexibility). ${ }^{56}$ The differences in the simulated hydrated electron properties must be due to the application of different electron-water molecule potentials. An additional motivation for the direct comparison of these two potentials is that both are developed on the same theoretical footing applying one-electron static-exchange (SE) pseudopotential theory. To further facilitate the present analysis, we will also perform simulations with a slightly modified version of the LGS model (m-LGS) with a polarization parameter that is similar to that in the TB model (see details later). We also note that we recently published a brief comparison of electron hydration dynamics in water clusters using the TB and LGS models. ${ }^{57}$ In that study we 
found a unique dynamical signature that is associated with the cavity collapse following electron removal from water cluster anions in the TB model (but not in LGS simulations). We suggested that this effect may be observed experimentally. ${ }^{57}$ The aim of the present paper is to provide a more general analysis and comparison. In this context we are aware that pseudopotentials, that treat solvent polarization explicitly, have been developed and published recently (the $\mathrm{JH}$ potential $^{42}$ and the self-consistent polarization potential of the Jordan group ${ }^{58}$ ). Acknowledging the merits of these models, for the reasons discussed above we omit them from the present comparison.

The structure of the paper will be as follows. In Sec. II, we briefly compare and analyze the main features of the two pseudopotentials based on a simple quantum mechanical model. Sec III collects the QCMD simulation results followed by a comparison of the predictions of the three different water molecule-electron pseudopotential models. Sec IV provides a discussion, and concludes the paper.

\section{The pseudopotentials}

The development of both pseudopotential models was based on the one-electron static exchange (SE) pseudopotential approximation. ${ }^{59}$ In the SE procedure, one solves the Schrödinger equation for an excess electron in the field of the frozen wave function of a single water molecule: $:^{39}$

$$
\left.H|\Psi\rangle=\left[T+V_{n}+V_{e}+V_{x}+V_{r}\right] \Psi\right\rangle
$$

In Eq. (1) $T$ is the kinetic energy operator, $V_{\mathrm{n}}, V_{\mathrm{e}}$ and $V_{\mathrm{x}}$ are the static nuclear attraction, the static electronic Coulomb repulsion and the non-local exchange, respectively. The repulsion 
operator $\left(V_{\mathrm{r}}\right)$ is introduced in Eq. (1) to account for the orthogonality of the core orbitals of the water molecule and the wave function of the excess electron. Following the Phillips-Kleinman theorem ${ }^{60}$ and the work of Cohen and Heine, ${ }^{61}$ it is then possible to solve Eq. (1) and obtain the exact pseudo-wave function, $\Psi$. In practice, this is performed by a minimization procedure of the kinetic energy of the excess electron with respect to small linear coefficients of the core orbitals $\left(\varphi_{\mathrm{c}}\right)$ that are mixed with the $\varphi_{\mathrm{v}}$ valence orbital (LUMO) of the water molecule. ${ }^{41}$

$$
|\Psi\rangle=\left(\left|\varphi_{v}\right\rangle+\sum_{c} b_{c}\left|\varphi_{c}\right\rangle\right) /\left(1+\sum_{c}\left|b_{c}\right|^{2}\right)
$$

Both the TB and LGS pseudopotentials used this procedure to receive $\Psi$, but the actual development of the potentials was different. TB introduced a smooth potential that reproduced the electron density of the pseudo-wave function as closely as possible (by minimizing the difference between the exact density and the density predicted by the given potential) ${ }^{41}$ while LGS directly constructed the pseudopotential from the pseudo-wave function and smoothed and fitted this "exact" potential afterwards. ${ }^{43,62}$

The different fitting procedure has important physical consequences. In the TB approach one can constrain the parameter fitting of the potential to solutions that closely preserve the eigenvalue of the SE Schrödinger equation (Eq. 1). The direct fitting of the exact potential by LGS, however, overlooked this basic requirement of the pseudopotential theory. Thus, although the fitted analytical function in the LGS model is apparently more flexible containing 16 optimized parameters of 8 Gaussian type functions, while the TB model applies only 4 error functions with 8 parameters, it is the TB model that reproduces the SE eigenvalue of the water molecule + electron model system. 
Before comparing the eigenvalues and wave functions of the two pseudopotentials, the quantum mechanical model, a water molecule and an excess electron evaluated within the SE approach, also needs a further consideration. ${ }^{63}$ Since a single water molecule is not able to bind an excess electron, it is only the limited size of the basis set that keeps the electron in the vicinity of the water molecule in finite basis calculations. The application of large diffuse basis functions (such that are used to generate the pseudo-wave function in the development of the two pseudopotentials) leads to a delocalized excess electron distribution, mainly outside the water molecule. The excess electron density in the chemically relevant region, in the vicinity of the water molecule, becomes extremely depleted. This diffuse electron distribution with very small amplitude in the core region makes it difficult (and uncertain) to precisely determine the exact underlying potential, as we will see below. This problem may be avoided with the application of a repulsive confining potential that artificially concentrates the excess electron density in the relevant molecular region, as we proposed some time ago: ${ }^{63}$

$$
V_{\text {conf }}=\frac{1}{2} k\left(x^{8}+y^{8}+z^{8}\right) \text {. }
$$

The potential was placed at the center of mass of the water molecule with $k=10^{-8}$ a.u. The steep repulsive walls and the flat central region of the potential ensure that the electron remains localized and the potential is not altered significantly around the water molecule. The effect of the confining potential on the exact pseudo-wave function is clearly illustrated in Figures 1 and 2 of Ref. 63 showing two orders of magnitude difference in the confined vs. non-confined solutions. While the TB potential was developed using a confining potential, LGS did not use this trick. For a direct comparison we performed the SE calculations on both pseudopotentials with and without the confining potential with the computational details described in Ref. 45. 
First, we start with the exact solution of the Schrödinger equation. The exact ground state eigenvalue of an unbound excess electron in the presence of the frozen water molecule wave function is $1.1973 \times 10^{-3}$ hartee. The energy of the electron, evaluated with the same finite basis set, becomes $1.3596 \times 10^{-3}$ hartree in the absence of the water molecule. This indicates that overall the water molecule slightly attracts the excess electron. The ground state energy of the exact model using the confining potential around the water molecule changes to $5.0257 \times 10^{-2}$ hartree. $^{45}$ Comparison with the ground state energy of the free electron in the confining potential $\left(4.6585 \times 10^{-2}\right.$ hartree) indicates that the potential in the vicinity of the water molecule is overall repulsive. ${ }^{45}$ The net attraction in the non-confined case is then clearly due to the long-range regions of the exact potential outside the molecular core. Next, we solve the Schrödinger equation for the excess electron in the field of the pseudopotential with and without the confining potential. This step provides a direct test of the performance of the pseudopotential models. While using the TB potential results in $5.0282 \times 10^{-2}$ hartree ground state energy for the excess electron in the confined case, in excellent agreement with the above exact number, the LGS potential shows significant deviation with $4.5725 \times 10^{-2}$ hartree..$^{45}$ For the non-confined problem that tests the asymptotic behavior, one receives $1.1829 \times 10^{-3}$ hartree with the TB model, and $1.2298 \times 10^{-3}$ hartree with the LGS potential. Here we now see some deviation for both models, but the TB model clearly performs better ( $\sim 1 \%$ error). For the LGS method, the confined eigenvalue is $\sim 9 \%$ too low (attractive), the non-confined is $\sim 3 \%$ too high (repulsive), showing that neither the core properties, nor the asymptotic behavior (although to a lesser extent) are satisfactory. Since the electron is expected to localize near the water molecules in condensed phase simulations, the results for the confined case are especially disturbing. Here we clearly 
observe that the LGS model replaces the originally overall repulsive exact potential to a (slightly) attractive interaction (relative to the free electron model in the confining potential). ${ }^{45}$

The excess electron density around the water molecule reflect the properties of the underlying pseudopotentials. Here we show projections of the electron density of the pseudowave function, and the densities computed with the TB and LGS models in the molecular plane along the dipole and the $\mathrm{OH}$ bond directions (Figure 1). At the outset, we observe that both potentials represent approximations to the exact pseudo density. Nevertheless, it is clear that the TB model does reproduce the asymptotic decay of the exact solution, while the LGS model fails in this respect. Looking at the core behavior, the LGS model does a better job at the vicinity of the hydrogen atoms, while the TB model is more reasonable in the dipole direction. We also note that in both density projections, the LGS model predicts significant increase at the oxygen side of the molecule. This feature is due to the fact that an attractive potential region appears behind the oxygen that we find to be inconsistent with basic chemical intuition. ${ }^{45}$ The artificial LGS density increase at the oxygen side is associated with a depletion of the density at the hydrogen side of the molecule, as clearly seen in Figure 1. Here one also has to make an additional point regarding the relation of the exact pseudo-wave function and the LGS potential development procedure. Larsen et al. claim to generate the exact potential and fit this potential with a suitable set of functions. ${ }^{43,62}$ The significant difference between the exact density and the LGS density clearly points to possible inconsistencies in the procedure.

Both the LGS and the TB model applies a polarization potential that is added to the SE potential a posteriori, in an ad hoc manner. ${ }^{41,43}$ While LGS use ${ }^{43}$ the polarization potential of Schnitker and Rossky, ${ }^{39}$ 


$$
V_{p o l}=-\frac{\alpha}{2 r^{4}}\left(1-\exp \left[-\left(r / r_{c}\right)^{6}\right]\right)
$$

TB apply ${ }^{41}$ the polarization of Barnett et al. ${ }^{40}$

$$
V_{p o l}=-\frac{\alpha}{2\left(r^{2}+C^{2}\right)^{2}}
$$

In Eqs (4) and (5) $\alpha$ is the spherical polarizability of the water molecule (9.7446 a.u.), $r$ is the electron-oxygen site distance, $r_{\mathrm{c}}=1.53 \AA$ and $C=4.4$ a.u. are cutoff distances. ${ }^{39,41,43}$ Since the LGS potential predicts a too deep ground state energy for the hydrated electron, ${ }^{43,45}$ in the present paper we attempt to weaken the polarization potential, similar to that used in the TB potential:

$$
V_{p o l}=-\frac{\alpha}{2\left(r^{2}+C^{2}\right)^{2}}\left(1-\exp \left[-\left(r / r_{c}\right)^{6}\right]\right)
$$

The modified LGS potential with the polarization of Eq. (6) will be denoted m-LGS in the remaining of the paper. The static exchange part of the LGS and TB potentials are shown in the dipole direction and along the $\mathrm{OH}$ bond in Figure 2. Figure 2 also contains the polarization potential for the three investigated models.

\section{Simulation details and results}

The simulations have been performed using a mixed quantum classical molecular dynamics method ${ }^{22}$ that has been developed and successfully employed in the Rossky group (see references in Ref 16). Since this technique has also been used by us previously in a series of studies, ${ }^{15,41,45,53,54}$ we focus only on its main features here. In the QCMD framework, the interaction between the classical water molecules is given by a flexible three-site potential $(\mathrm{SPC}+\mathrm{flex}),{ }^{56}$ while the excess electron is described by its wave function. The wave function is 
represented on a finite grid, evenly distributed in a cubic box. For the TB potential we use a cubic grid with a box side of $18.17 \AA$ with $16 \times 16 \times 16$ equidistant grid points. Due to the more rugged features of the potential surface, the evaluation of the LGS potential requires denser spatial grid. For this, a grid box of $18.17 \AA$ length with $32 \times 32 \times 32$ grid points proved to be sufficient. The electron-water molecule interaction is modeled by a pseudopotential, either the $\mathrm{TB}^{41}$ potential or the LGS potential. ${ }^{43}$ The time-independent Schrödinger equation for the excess electron is solved in the potential field of the classical water molecules using an iterative and block Lanczos procedure. $^{22}$ The time evolution of the classical subsystem is dictated by the sum of a quantum force (from the electron) and a classical force (from the other molecules). The quantum force is evaluated applying the Hellman-Feynman theorem. The dynamics is adiabatic. We use the Verlet algorithm to integrate the equations of motion with a time step of $1 \mathrm{fs}^{64}$ The simulated water clusters contain $n=45,66,104,200,500$ and 1000 water molecules. The simulations were carried out in the microcanonical ensemble. The trajectories are equilibrated at two different effective temperatures, at $200 \mathrm{~K}$ and $298 \mathrm{~K}$. The former is used to compare the simulation results to low temperature cluster measurements, while the $298 \mathrm{~K}$ data are used for the extrapolation to bulk hydrated electron properties at ambient conditions. The analysis of the results is based on 50 ps long equilibrium trajectories for all cluster sizes, pseudopotentials and temperatures.

First, we examine the structural properties of the hydrated electron clusters. The first observation concerns the position of the equilibrated electron relative to the molecular framework of the cluster (Figure 3). By using our previous simple criterion, an electron is localized in the interior of the cluster if $R+r_{\mathrm{e}}<r_{\mathrm{c}}$, where $r_{\mathrm{e}}$ is the radius of gyration of the excess electron $\left(r_{e}=\left\langle r^{2}\right\rangle^{1 / 2}\right), r_{\mathrm{c}}$ is the radius of the cluster, and $R$ is the distance between the centers (center-of- 
mass, com) of the two distributions. ${ }^{15,53}$ For surface-bound states, one has $R \sim r_{c}$. For the TB model we find these two main cluster types (Figure 3). Initial electron attachment to equilibrated neutral clusters and the following relaxation always result in surface state excess electron distributions at $200 \mathrm{~K} .{ }^{53,54}$ Trajectories at $200 \mathrm{~K}$ launched from neutral clusters with preformed cavities persist in interior state structures roughly for $n \geq 200$ cluster sizes for the typical length of such trajectories, several hundred ps. At higher temperature, $298 \mathrm{~K}$, while cluster anions relax in surface state after electron attachment for $n=45-200$, larger clusters ( $n=500,1000)$ show increased tendency for the electron to diffuse spontaneously into the interior of the cluster. ${ }^{53}$ The results are more monotonic for the LGS and the m-LGS models. For all cases, regardless of the size or the temperature of the clusters, excess electron initial localization and relaxation takes place in the interior of the cluster (Figure 3). Besides the electron attachment scenario to neutral clusters, we also tested electron localization with the LGS potential starting from configurations that supported surface states in the TB model (preformed surface state clusters). We found that all these clusters also collapse to interior state structures within less than $\sim 1$ ps using both the LGS and the m-LGS models. The contrast of the TB and LGS models is also illustrated via the geometrical parameters, as seen in Figure 4 for a relatively small cluster size, $n=104$, at $T=200$ K. At this size, the TB model predicts surface state isomer, while both LGS models prefer interior state as demonstrated by the $R$ parameter in Figure 4. As expected, the surface state electron (TB) is more diffuse than the interior state electron in LGS (see parameter $r_{\mathrm{e}}$, the radius of the excess electron in Figure 4). At larger cluster sizes, while interior state isomers may also appear using the TB model, the LGS models unanimously predict interior state electron distribution. We analyze the cluster size dependence of the size of the excess electron later. Here we note that the relative position of the excess electron to the cluster may also depend on the cluster preparation 
method, as was illustrated by one-electron pseudopotential (TB) ${ }^{65}$ and AIMD simulations. ${ }^{66}$ As an additional comparison, another one-electron pseudopotential $(\mathrm{JH})$ that simulates cavity structure for the bulk hydrated electron predicts four types of cluster anion isomers depending on cluster size, temperature and cluster preparation using a different criterion for the surface geometry. ${ }^{55,67}$

Next, we characterize the local water structure surrounding the electron. The solvent structure (around the solute), and the solute structure (the excess electron distribution in the water bath) are mutually determined via the electron-water molecule interaction. The LGS and TB models predict conceptually different excess electron states in the bulk, and different water structure in the vicinity of the electron. While the TB model results in cavity type structure, ${ }^{41}$ the LGS model simulates non-cavity electron distribution. ${ }^{43}$ The relative position of the electron distribution with respect to the cluster interfaces may further complicate the structural details of water cluster anions. The TB model does indeed fall in this category with its at least two isomeric water cluster anion groups. Figure 5 shows the electron com-oxygen site and the electron comhydrogen site radial distribution functions (rdf) for selected size clusters computed with the TB model at $200 \mathrm{~K}$. At this temperature both interior and surface excess electron states can be realized depending on the cluster size. For both types of isomers one observes an excluded volume around the electron in the distribution functions. The position of the first maxima appears rather uniformly at $\sim 2 \AA$ for the hydrogen rdf, and $\sim 3 \AA$ for the oxygen rdf, consistent with bond directed water orientation toward the electron, similar to that found in bulk. ${ }^{41}$ The similar position of the maxima, and the relative intensity of the rdf's for the interior state clusters relative to those of surface state clusters of the same size $(\sim 1.5-1.8$ for the $n=500,1000$ cases $)$ suggests that surface state excess electrons localize in dents on the surface, nearly in a half- 
cavity. As the clusters grow the dent gradually forms and becomes deeper, indicated by the increasing intensity of the first maximum of surface state clusters with size. We also find that smaller clusters have more diffuse radial distribution functions, consistent with weaker electroncluster interaction. Increasing the temperature (to $T=298 \mathrm{~K}$ ) does not alter the major tendencies, except in two aspects. The functions (not shown) become somewhat less structured at higher $T$, and the size of the excluded volume, although its presence is apparent, shrinks. We also note that since surface state electrons diffuse to the interior of the cluster for $n=500$ and 1000 clusters, direct comparison of interior state clusters vs. surface state clusters of the same size is not possible at $298 \mathrm{~K}$.

The structural predictions of the LGS model at $298 \mathrm{~K}$ for clusters strongly parallel the results found for the ambient bulk hydrated electron with the same model. ${ }^{43}$ No cavity is formed for the interior stabilized excess electron, as indicated by the apparent lack of the excluded volume in the pair correlation functions of the clusters (Figure 6). The maxima (approximately identical with the bulk $298 \mathrm{~K}$ data) appear at $\sim 1 \AA$ for the hydrogen and $\sim 2 \AA$ for the oxygen rdf's (not shown) irrespective of the cluster size. For the 200 K LGS cluster anions the sign of tiny cavities (with small excluded radius, $\sim 0.5 \AA$ ) becomes evident (Figure 6). This suggests that the water kinetic energy is not sufficient to collapse the cavity below ambient temperatures in the LGS model. The m-LGS model predicts significantly weaker interaction of the electron with the water molecules due to its scaled down polarization part (Eq. 6). Still, the m-LGS radial distribution functions are not very different from those computed with LGS. Interestingly, although in m-LGS the hydrogen radial distribution functions indicate non-cavity structure, the directional preference of the $\mathrm{OH}$ bonds toward the electron essentially disappears at $298 \mathrm{~K}$. At 
lower temperatures $(T=200 \mathrm{~K})$ both the cavity character and the $\mathrm{OH}$ directionality reappears in m-LGS simulations (Figure 6).

The next analyzed characteristic structural property is the radius of the electron. Using spectral moment analysis of absorption spectra Bartels derived experimental excess electron radius data for size selected water cluster anions. ${ }^{48}$ The data evaluation of Bartels suggests that as the cluster size grows, the size of the electron distribution shrinks approaching an asymptotic limit from above. We note, that although the experimentally examined clusters are smaller in size than the present simulated ones, one can expect that the observed trend continues for larger clusters, as well. It was shown previously that the experimental tendency is followed qualitatively by cold $(200 \mathrm{~K})$ surface state isomers in simulations using the TB model. ${ }^{15,53}$ Here we also point out that the size of surface state electrons is somewhat overestimated in the TB model. Interior state excess electrons of the TB model have essentially constant radius $(2.36 \AA$ for $n=500$ and 1000 interior state clusters at $298 \mathrm{~K}$ ), in good agreement with the simulated radius of the ambient bulk hydrated electron $(2.42 \AA) .{ }^{15,53}$ Similar overall tendencies are seen with another recent cavity predicting pseudopotential, the $\mathrm{JH}$ model, that is not examined here. ${ }^{42,55}$ Figure 7 collects the simulated radius data at $200 \mathrm{~K}$. The LGS model predicts only interior state clusters in the examined size range. At $200 \mathrm{~K}$ the radius shows essentially no size dependence ranging between 2.0 to $2.2 \AA$. At $298 \mathrm{~K}$ one observes a moderately increasing tendency from $2.42 \AA(n=45)$ to $2.64 \AA(n=1000)$. Although this latter value is consistent with the simulated LGS bulk hydrated electron radius, ${ }^{43}$ it overestimates the experimentally inferred radius $(2.45 \AA$ at ambient conditions). ${ }^{49}$ In addition, the LGS tendencies are clearly at odds with that found experimentally for the size dependence of the radius. ${ }^{48}$ Here, we also indicate that we also performed test simulations for clusters below the examined size range ( $n=20$ and 30 clusters). We found that the 
above tendencies definitely continue to lower cluster sizes, as well. Using a weaker polarization interaction in the m-LGS potential results in a radius pattern with size that resembles to the experimental tendency. The radii of cold clusters decrease with cluster size, and reach a radius (2.32 $\AA$ ) in the $n=1000$ cluster that compares favorably with the ambient bulk radius (Figure 7). At ambient conditions the clearly decreasing $200 \mathrm{~K}$ tendency disappears, and one observes a delocalized excess electron structure with $\sim 4 \AA$ radius in the largest examined clusters.

Now we turn our attention to the energetic and steady-state spectroscopic data of water cluster anions. The experimental VDE data for water cluster anions reflect characteristic trends with size, ${ }^{10,50}$ and reasonable models are expected to capture these qualitative features. Figure 8 contains the computed VDE data as a function of cluster size. Here, we use the usual $n^{-1 / 3}$ representation of the data that is used in dielectric continuum models, and leads to an apparent linear behavior. ${ }^{68}$ The TB model was previously shown to predict at least two different linear patterns. ${ }^{15,53}$ At $200 \mathrm{~K}$, all examined clusters were found to stabilize surface state excess electron distribution, but interior states were also simulated for clusters with $n \geq 500$. Both patterns were also located at a higher temperature $(298 \mathrm{~K})$, with surface states persisting up to $n$ 500, and larger clusters stabilizing only in interior state. Interestingly, the TB computed VDE values did not show significant temperature dependence in this temperature range. ${ }^{15,53}$ Linear extrapolation to $n^{-1 / 3}=0$ provides the simulated bulk limit for the hydrated electron VDE. Simulations for interior state excess electrons gave $4.4 \mathrm{eV}$, while surface state data extrapolate to $3.9 \mathrm{eV}$. Both values indicate stronger interaction than the $3.3 \mathrm{eV}$ value extrapolated from cluster measurements, ${ }^{49}$ and data from direct bulk VDE measurements $\left(3.3 \mathrm{eV},{ }^{7} 3.3 \mathrm{eV}^{69}\right.$ and $\left.3.6 \mathrm{eV}^{70}\right)$. Furthermore, very low temperature simulations suggest the possible formation of metastable cluster anions that may appear as another distinct group in the VDE - size diagram. ${ }^{65,66}$ 
LGS simulations, however, show no sign of the existence of distinct cluster anion isomers for $n \geq 45$ at the examined $200 \mathrm{~K}$ and $298 \mathrm{~K}$ temperatures. ${ }^{71}$ In addition, for the only localized isomer type, the interior state isomer, the LGS model predicts significantly stronger binding energy than the experimental VDE range and the TB potential. For example, the VDE of the $n=$ 1000 cluster anion with interior excess electron is $\sim 3.5 \mathrm{eV}$ in $\mathrm{TB}$ calculations, while it is $\sim 6.5 \mathrm{eV}$ and $\sim 6 \mathrm{eV}$ with the LGS model at $200 \mathrm{~K}$ and $298 \mathrm{~K}$, respectively (Figure 8). Clearly, temperature appears to be an important factor, the $200 \mathrm{~K} \mathrm{VDE}$ data being significantly greater (by $\sim 0.6 \mathrm{eV}$ for the largest clusters) than the $298 \mathrm{~K}$ VDE. So, although only a single structural isomer type appears in LGS simulations, two patterns of VDE is observable with size, due to a temperature effect. It is also notable that the $n^{-1 / 3}$ representation of the data approximately follows linearity. Linear extrapolation to the infinite size asymptotic limit shows unrealistically high $\sim 8 \mathrm{eV}$ and $\sim 7$ eV bulk VDE values at $200 \mathrm{~K}$ and $298 \mathrm{~K}$. Weakening the polarization part of the LGS potential to that similar to the TB potential scales down the VDE values by a factor of $\sim 2$, placing them closer to, but not quite in the experimental data range. The linear character of the VDE $-n^{-1 / 3}$ function is again approximately correct with the estimated asymptotic values of $\sim 5.5 \mathrm{eV}$ and $\sim 4$ $\mathrm{eV}$ at $200 \mathrm{~K}$ and $298 \mathrm{~K}$, respectively.

Finally, we also calculated the optical absorption spectra for the simulated cluster anions. Experimentally the spectra shift to higher energies with increasing cluster size, apparently extrapolating to a bulk limit. ${ }^{72}$ This tendency was illustrated to appear in the surface state isomers using the TB model, while for interior state clusters the same model predicts essentially sizeindependent position of the spectra. ${ }^{15,53}$ With the LGS model at $298 \mathrm{~K}$ one observes that smaller clusters have a spectral maximum at $\sim 1.8 \mathrm{eV}(n=45,66)$, while larger clusters appear $(n=500$, 1000) to be slightly red-shifted to $\sim 1.5 \mathrm{eV}$. This latter estimate is consistent with LGS bulk 
simulation results, ${ }^{43}$ thus underestimates the experimental position by $\sim 0.2 \mathrm{eV} .{ }^{73}$ Similar simulations at $200 \mathrm{~K}$ shift the spectra significantly to higher energies, between $\sim 1.9 \mathrm{eV}$ and $\sim 2.3$ eV. Apparently, no clear size-dependent trend is seen on the evolution of the spectra at either temperatures with the LGS potential. The m-LGS model at $200 \mathrm{~K}$ predicts very similar tendency to the surface state TB results and the experiment with a clear shift of the position of the spectral maximum from $0.9 \mathrm{eV}(n=45)$ to $1.7 \mathrm{eV}(n=1000)$. The m-LGS $298 \mathrm{~K}$ simulations, however, appear to take an opposite trend progressing from $0.8 \mathrm{eV}(n=45)$ to $0.5 \mathrm{eV}(n=1000)$. The $298 \mathrm{~K}$ tendency seems to be completely unphysical, and discredits the otherwise promising tendency at $200 \mathrm{~K}$.

\section{Discussion and Conclusions}

We examined and compared three electron-water molecule pseudopotential models. The TB and LGS potentials are both developed using the static exchange theorem with the polarization part added a posteriori. Despite the similar potential development procedure, the two potentials lead to dramatically different physical properties of the hydrated electron. Most notably, while TB model supports the traditional cavity picture of the hydrated electron, the LGS model predicts a non-cavity structure with increased local water density in the vicinity of the excess electron. To separate the effect of the different polarization contributions in the two models, we also examine the LGS potential with a modified polarization (m-LGS potential) that is weakened to that used in the TB model. The present analysis focuses on two main aspects: a) quantum calculations on a model system consisting one water molecule and an excess electron, and b) adiabatic mixed quantum-classical molecular dynamics simulations on various size water cluster anions using the different pseudopotential models. 
The exact quantum mechanical calculations clearly demonstrate an inherent contradiction in the LGS potential development procedure. The exact LGS potential mapping followed by potential fitting leads to inaccurate numerical results in model calculations. Here the LGS model apparently fails to satisfy the most basic requirements of pseudopotential theory: reproduce the exact eigenvalue of the excess electronic state of the model and the asymptotic behavior of its wave function. In particular, we observe that the LGS potential is overall too attractive, in particular around the oxygen core with the appearance of an unexpected attractive region on the oxygen side of the molecule. The TB potential has been shown to predict better agreement with model calculations. ${ }^{15,53}$

Mixed quantum-classical molecular dynamics simulations of water cluster anions using different pseudopotential models were also carried out for further testing purposes. Since direct experimental data exist for bulk hydrated electron and hydrated electron clusters, comparison with experiments are used to assess the viability of the potentials. The TB model performs in a semi-quantitative manner with respect to experimental cluster geometric, energetic and spectroscopic data. The potential predicts at least two structural isomers for water cluster anions, interior state and surface state clusters. The trends of the radius of gyration, the vertical detachment energies and the absorption spectra with size reproduce the experimentally observed tendencies. This set of findings has served as a basis for the assignment of the experimental type I clusters as surface state clusters. ${ }^{15,53}$

The LGS potential predicts the presence of only one isomer type, interior state clusters. The radius of gyration of the interior state clusters is similar to that found for the interior state clusters simulated with the TB model at $200 \mathrm{~K}$. In addition, the radius of the interior excess electron is basically independent of the cluster size in cold clusters. At ambient temperature, the 
size dependence of the LGS clusters is opposite to the observed experimental tendency, and the electron becomes more diffuse with increasing cluster size. The different tendencies at different temperatures reflect the structural changes of the solvent in the vicinity of the electron. At low temperatures one observes formation of a cavity type solvation structure, while at higher $T$ the structure collapses to the non-cavity type arrangement. LGS simulations also predict a significant overbinding of the excess electron, the VDE values being a factor of $\sim 2$ larger than the experimental data. The VDE does depend on the temperature, the low temperature cavity forming clusters bind the electron $\sim 1 \mathrm{eV}$ stronger than the ambient non-cavity clusters. The position of the maxima of the absorption spectra with size also appears to be in disagreement with experiment. While small cluster experimental spectra show up in the low energy part of the spectrum $(\sim 1 \mathrm{eV})$, all LGS cluster absorption spectra appears at around or beyond the experimental $1.72 \mathrm{eV}$ bulk spectrum at both examined temperatures.

Weakening the polarization part of the m-LGS potential illustrates that most of the unusual properties of the LGS model originates from the static exchange part of the potential. Most importantly the non-cavity structure persist in the m-LGS simulations at $298 \mathrm{~K}$, and the decrease of temperature leads again to the reappearance of a small cavity. The overbinding decreases with the scaled down polarization, but one still observes a single interior state isomer type of the cluster anions. The evolution of the radius of the electron with size now resembles the experimental tendency in cold m-LGS clusters, but then the $298 \mathrm{~K}$ data become absurdly large. Similarly, while the positions of the m-LGS simulated $200 \mathrm{~K}$ spectra correspond well to the experimental trend, the $298 \mathrm{~K}$ spectra turn out to be nonsensical. We conclude that modification of the polarization part of the LGS potential is not likely to improve the LGS results significantly. 
All in all, our analysis suggests that the pseudopotential model of Larsen et al. that results in non-cavity structure in bulk hydrated electron simulations, predicts cluster anion properties that are, in most of the analyzed cases, not in line with experimental observations. The cavity type TB model that were used for comparison fares much better. It is not to say that the analysis proves anything about the structure of the hydrated electron. We simply state, that within the limitations of the models, the cavity preferring model gives significantly better agreement with cluster experiments than the examined non-cavity predicting LGS model. In terms of the cavity vs. non-cavity debate, it may be of interest to reiterate here the recent AIMD simulation work of Jungwirth et el. who found clear signature of the cavity structure in their extended bulk simulations. Although their predicted excluded volume is somewhat smaller than in the TB model, the message of the existence of the excluded volume is clear. ${ }^{28}$

\section{Acknowledgments}

This work was supported by a research grant to LT from the National Research Fund of Hungary (OTKA, K104237). 


\section{Figure Captions}

Figure 1. The electron density of the exact pseudo-wave function (black), the LGS electron density (blue), and the TB electron density (red) along the dipole direction in the molecular plane through the oxygen atom (top) and along the $\mathrm{OH}$ bond (bottom). The center of mass of the water molecule is at the origin; the hydrogen atoms are at negative coordinates.

Figure 2. The static exchange potential of the LGS potential (blue), and the TB potential (red) along the dipole direction in the molecular plane through the oxygen atom (top) and along the $\mathrm{OH}$ bond (middle). The lower panel show the polarization contributions to the full potential for the LGS (blue), TB (red) and m-LGS (blue dashed) models. The origin for the dipole direction is at the center of mass of the water molecule (top), the oxygen atom for the $\mathrm{OH}$ direction (middle) and the polarization potential (bottom). The hydrogen atoms are at negative coordinates.

Figure 3. Electron density isosurfaces of the excess electron in water cluster anions as calculated with the LGS (top) and the TB (bottom) models at $200 \mathrm{~K}$. The isosurfaces show $80 \%$ probability of finding the excess electron.

Figure 4. Equilibrium time evolution of the radius of electron $\left(r_{\mathrm{e}}\right)$, the radius of the cluster $\left(r_{\mathrm{c}}\right)$ and the distance between the com of the water cluster and the com of the excess electron $(R)$ at $T=200 \mathrm{~K}$ in $n=104$ water cluster anions. Red shows the results using the LGS model, blue indicates TB simulations.

Figure 5. Electron com-oxygen and electron com-hydrogen radial distribution functions simulated with the TB model at $200 \mathrm{~K}$ in water cluster anions. The cluster sizes are $n=45$ (magenta), 66 (cyan), 104 (blue), 200 (green), 500 (red) and 1000 (black). Surface state clusters are indicated by solid lines, interior states by dashed lines. 
Figure 6. Electron com-hydrogen radial distribution functions simulated with the LGS and the m-LGS models at $200 \mathrm{~K}$ and $298 \mathrm{~K}$ in water cluster anions. The cluster sizes are $n=45$ (magenta), 66 (cyan), 104 (blue), 200 (green), 500 (red) and 1000 (black). All clusters are interior state clusters.

Figure 7. Radius of gyration of the excess electron in various size water cluster anions computed at $200 \mathrm{~K}$ using the TB model ( $\bullet$ ), the LGS model $(\bullet)$ and the modified m-LGS model ( $\boldsymbol{\Delta})$. Open squares show surface state cluster anions, realized only in the TB model ( $\square$ ).

Figure 8. Vertical detachment energy of the excess electron in various size water cluster anions computed using the TB model, the LGS model and m-LGS model at $200 \mathrm{~K}$ (full symbols) and 298 K (open symbols). Squares show interior state cluster anions, triangles indicate surface state anions. 
Figure 1.

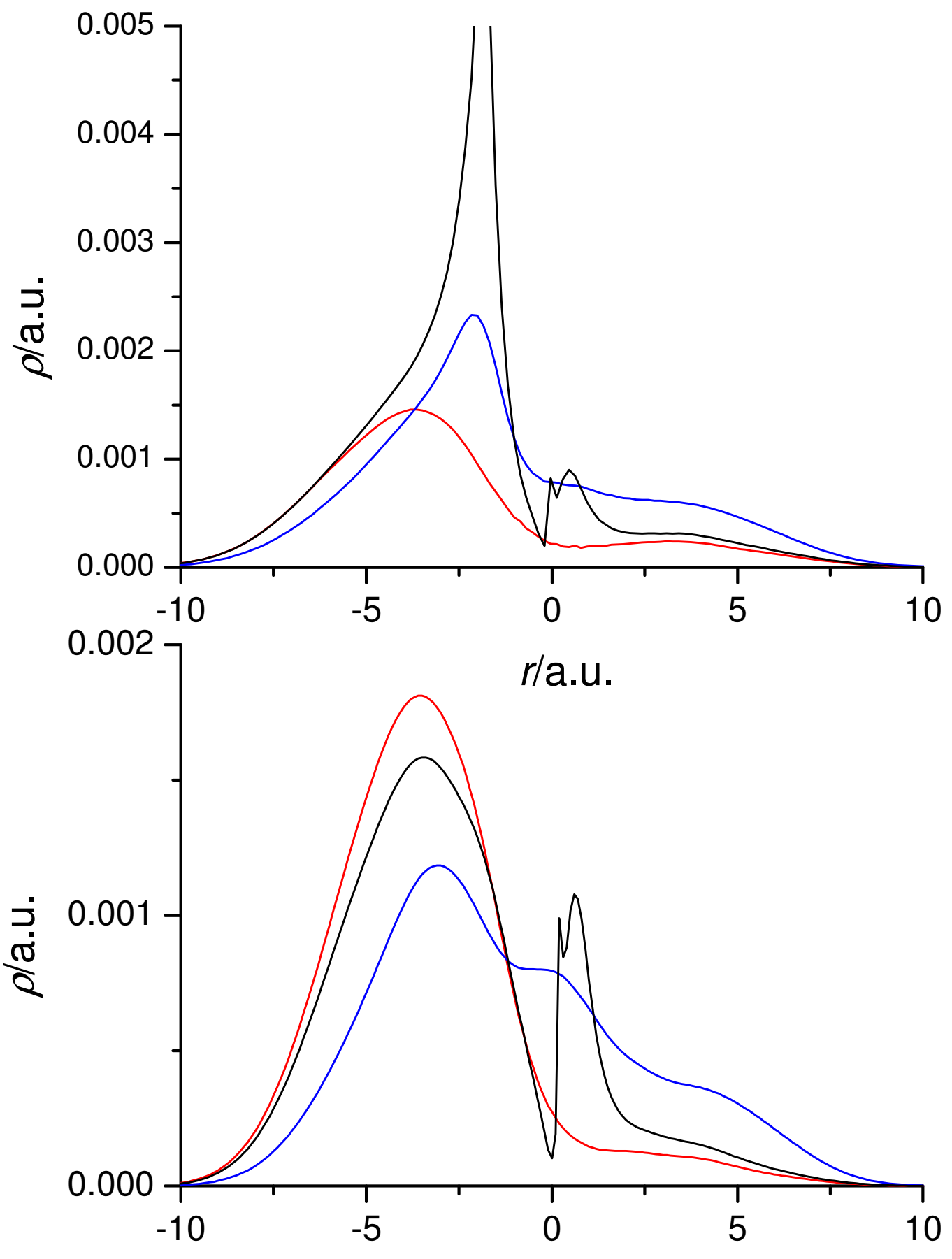


Figure 2.
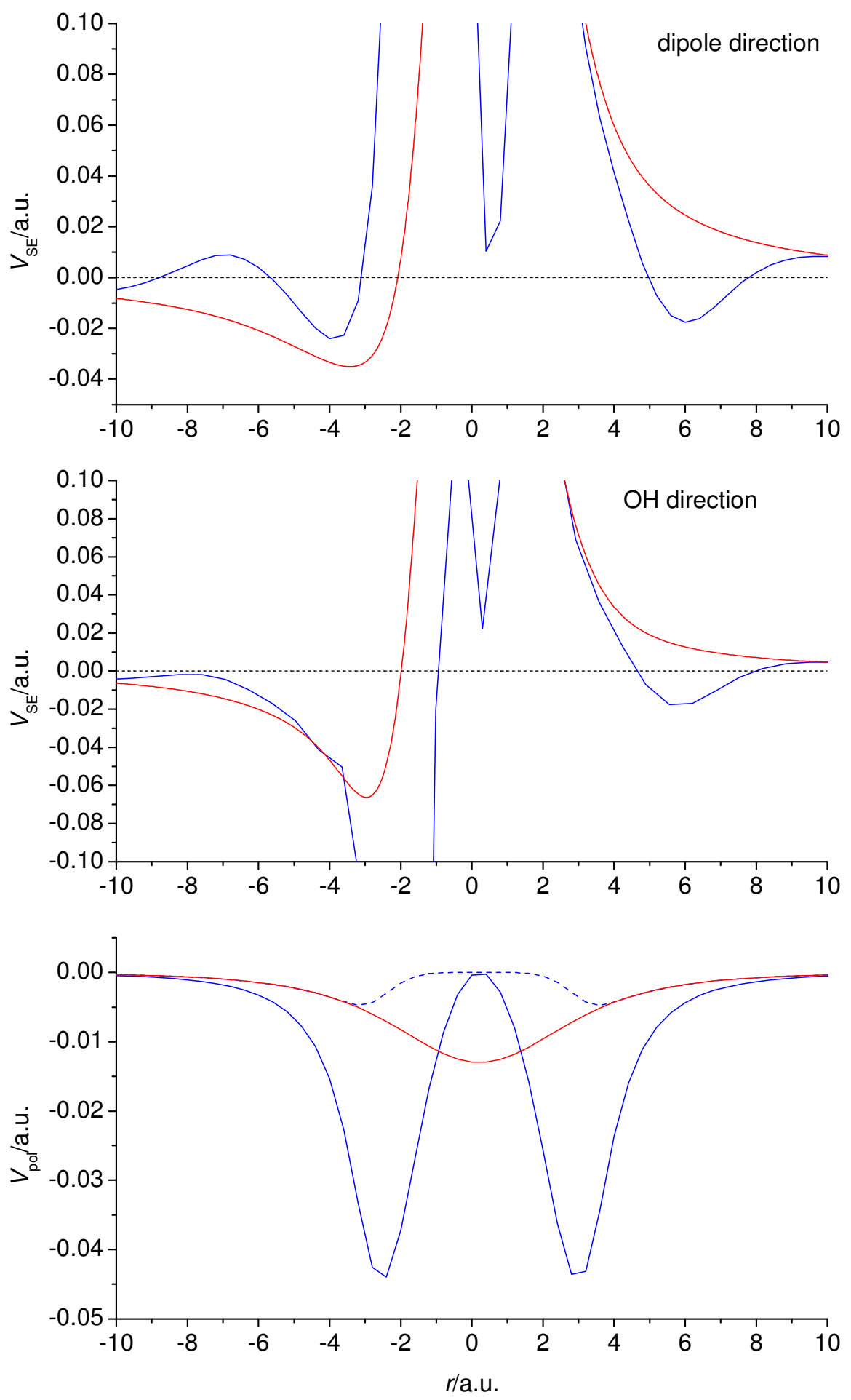
Figure 3.

\section{LGS potential}
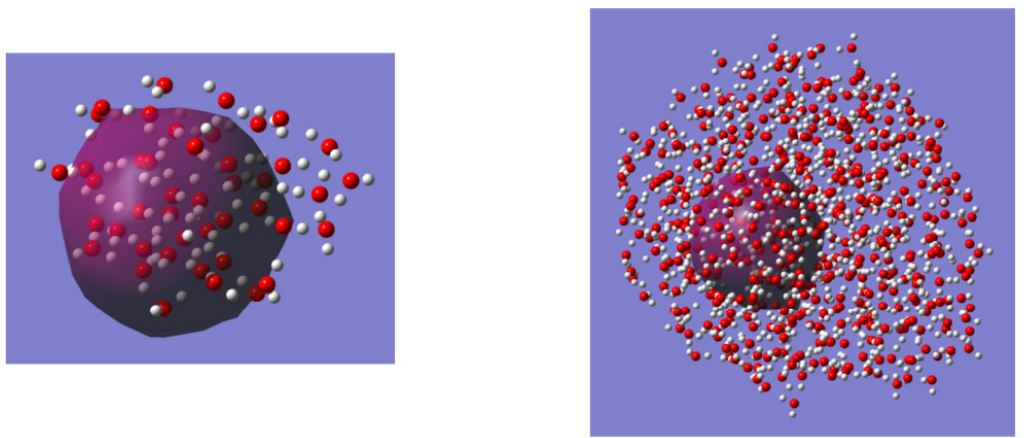

$n=45$, interior

$n=500$, interior

TB potential

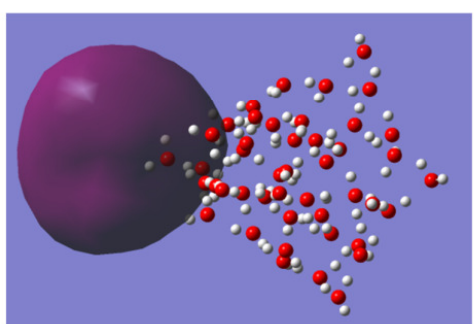

$n=45$, surface

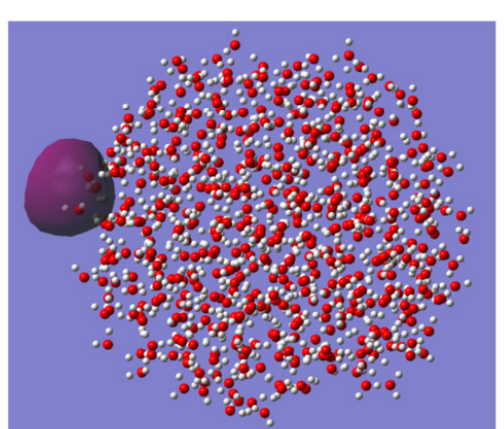

$n=500$, surface

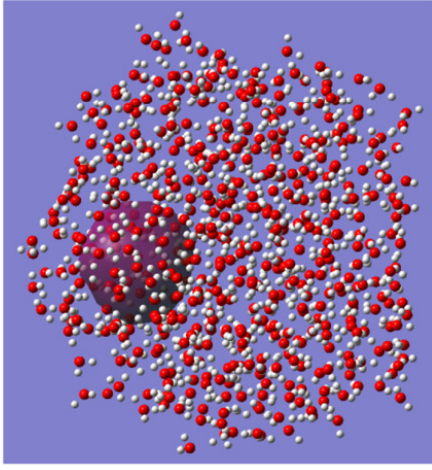

$n=500$, interior 
Figure 4.

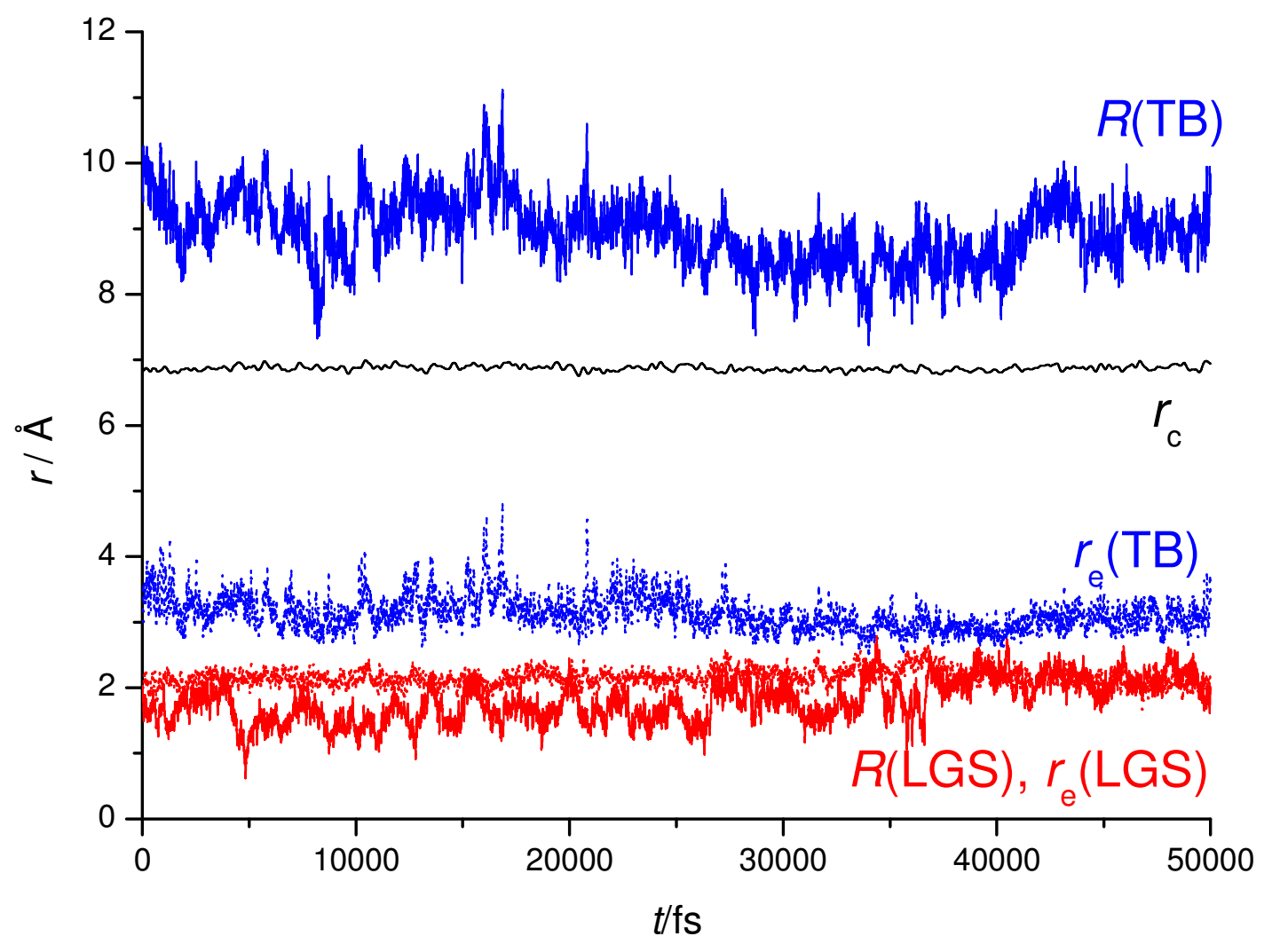


Figure 5.
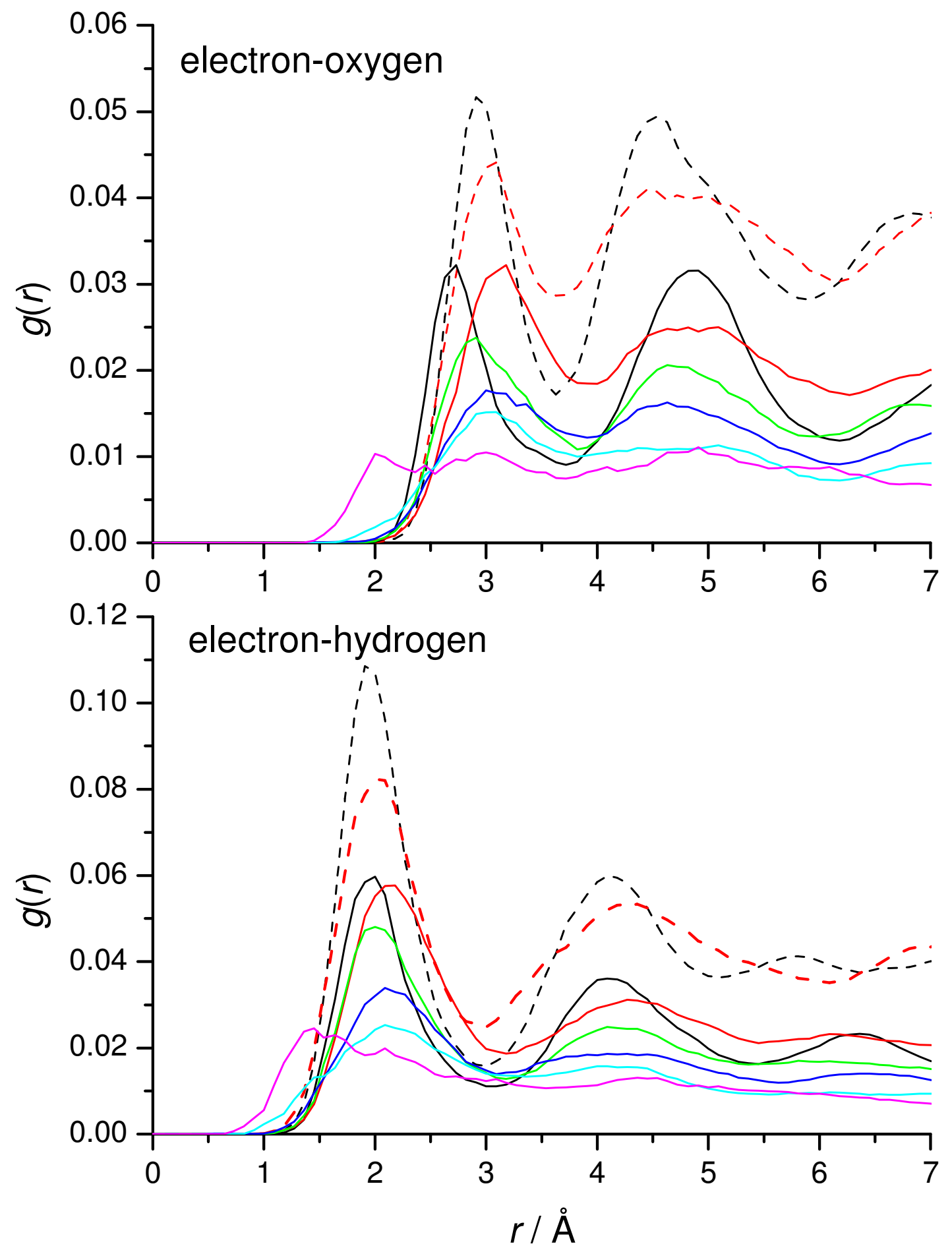
Figure 6.
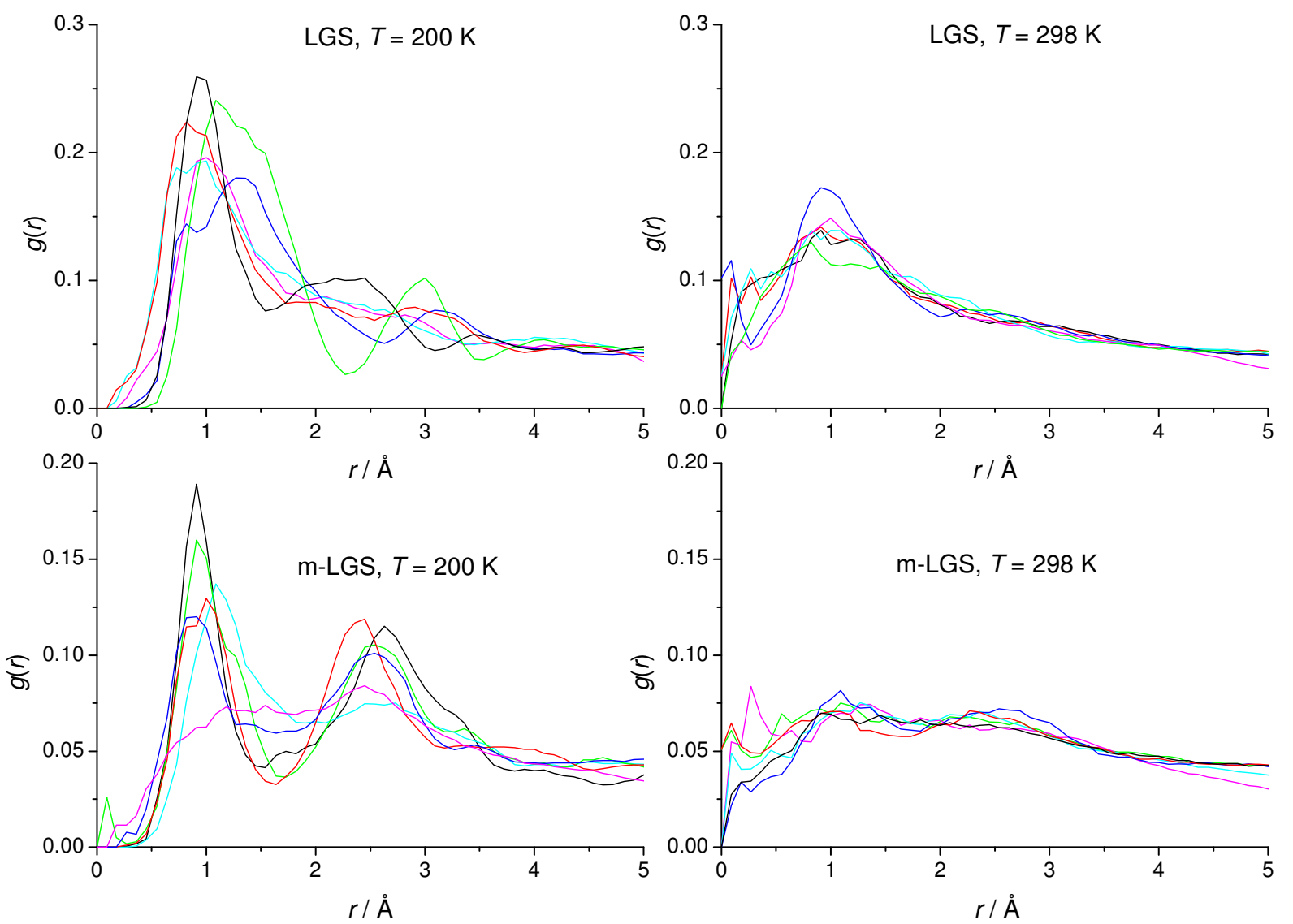
Figure 7.

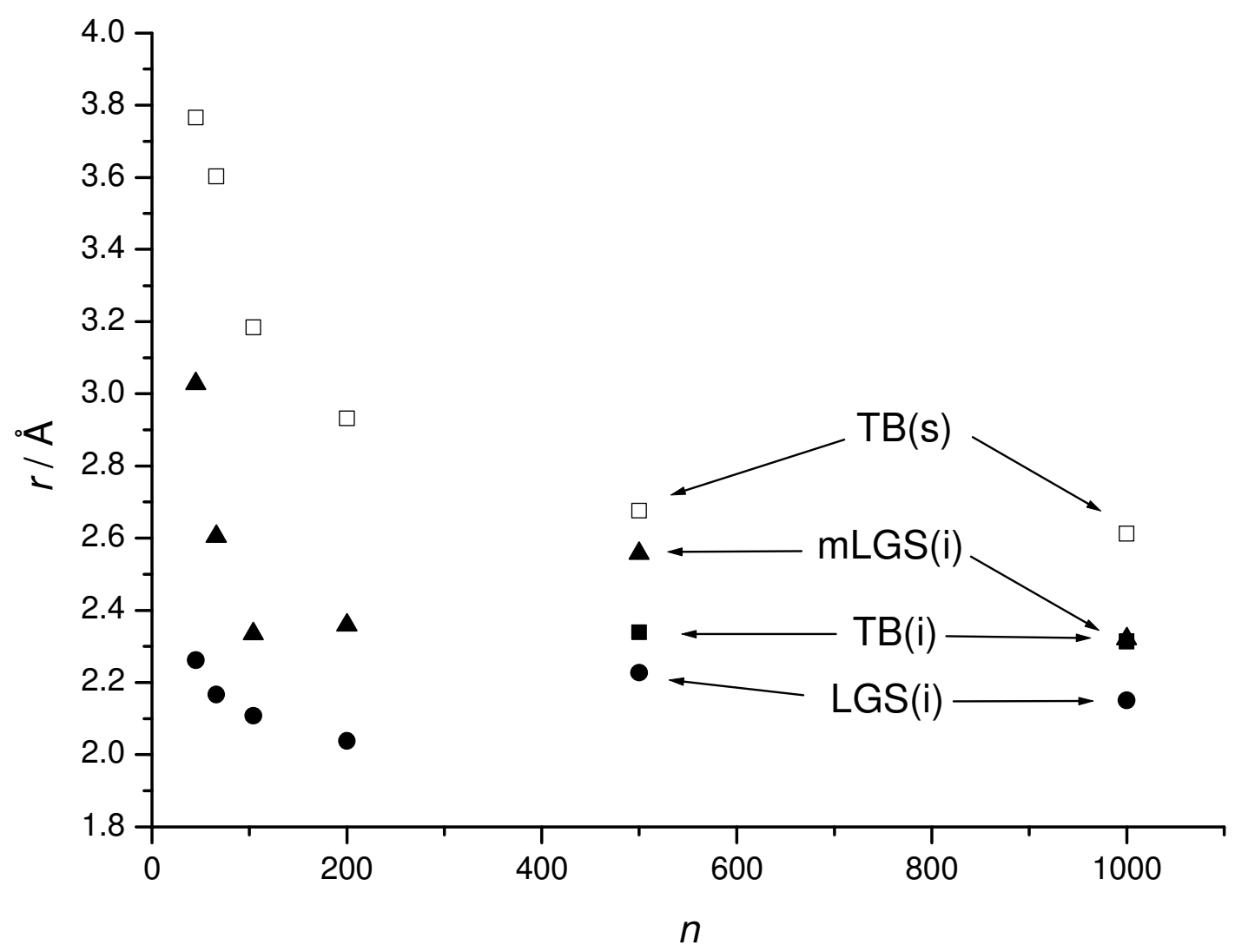


Figure 8.
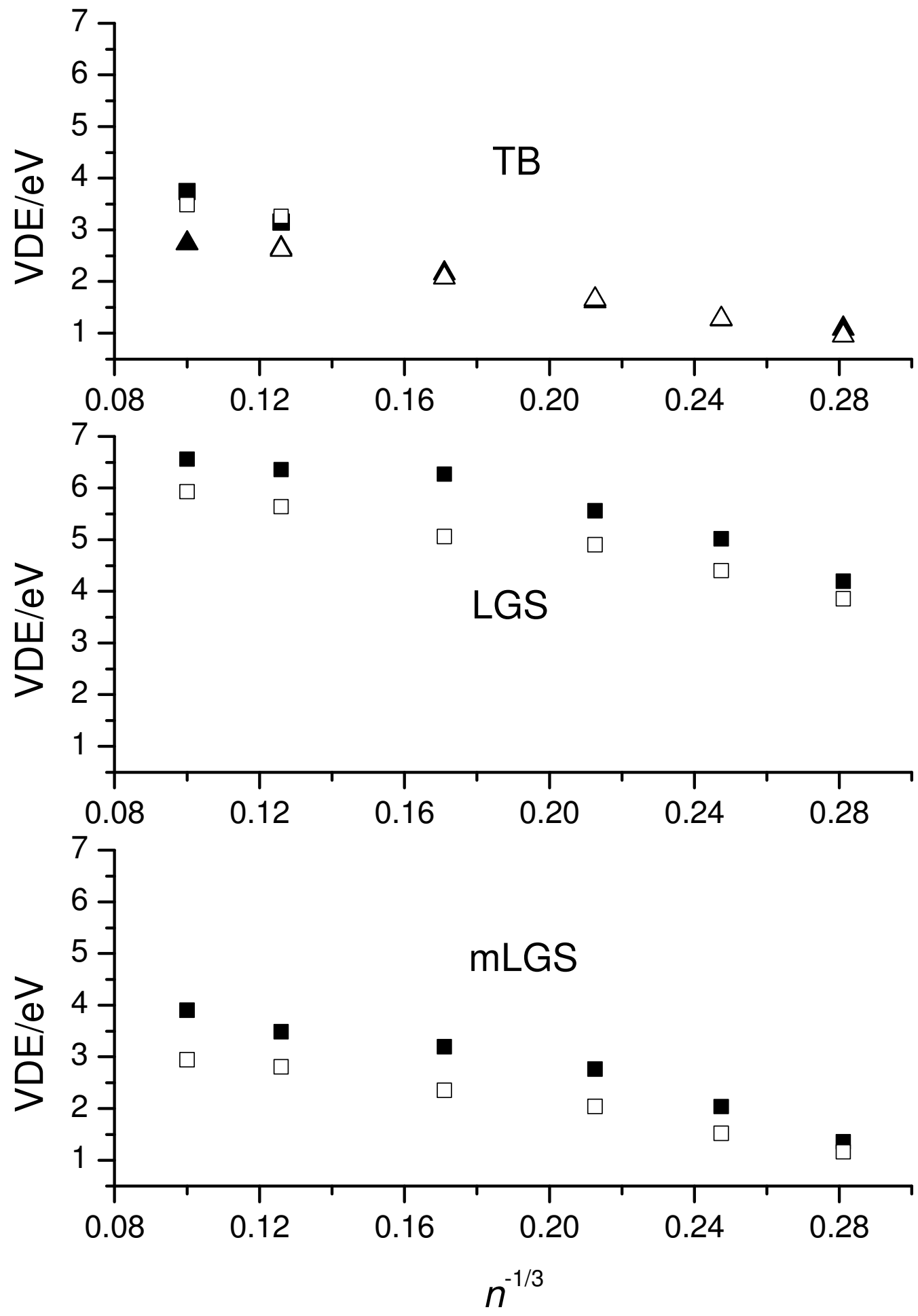


\section{for Table of Contents use only}

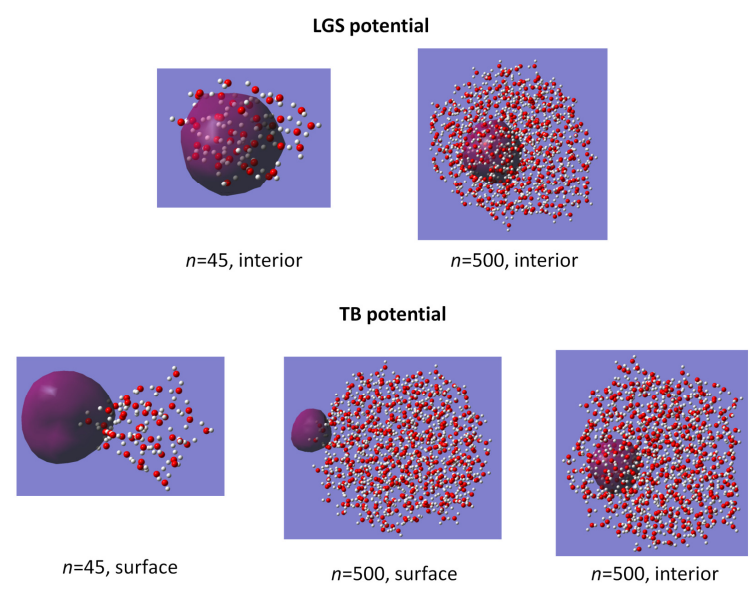




\section{References}

(1) Hart, E. J.; Boag, J. W. J. Am. Chem. Soc. 1962, 84, 4090.

(2 ) Hart, E. J.; Anbar, M. The Hydrated Electron, Wiley-Interscience: New York, NY, 1970.

(3) Garrett, B. C.; Dixon, D. A.; Camaioni, D. M.; Chipman, D. M.; Johnson, M. A.; Jonah,

C. D.; Kimmel, G. A.; Miller, J. H.; Rescigno, T. N.; Rossky, P. J.; Xantheas, S. S.; Colson, S. D.; Laufer, A. H.; Ray, D.; Barbara, P. F.; Bartels, D. M.; Becker, K. H.; Bowen, H.;

Bradforth, S. E.; Carmichael, I.; Coe, J. V.; Corrales, L. R.; Cowin, J. P.; Dupuis, M.;

Eisenthal, K. B.; Franz, J. A.; Gutowski, M. S.; Jordan, K. D.; Kay, B. D.; LaVerne, J. A.; Lymar, S. V.; Madey, T. E.; McCurdy, C. W.; Meisel, D.; Mukamel, S.; Nilsson, A. R.;

Orlando, T. M.; Petrik, N. G.; Pimblott, S. M.; Rustad, J. R.; Schenter, G. K.; Singer, S. J.;

Tokmakoff, A.; Wang, L.-S.; Wittig, C.; Zwier, T. S. Chem. Rev. 2005, 105, 355.

(4) Elkins, M. H.; Williams, H. L.; Shreve, A. T.; Neumark, D. M. Science 2013, 342, 6165.

(5) Buchner, F.; Schultz, T.; Lübcke A. Phys. Chem. Chem. Phys. 2012, 14, 5837.

(6) Siefermann, K. R.; Abel, B. Angew. Chem. Int. Ed. 2011, 50, 5264.

(7) Siefermann, K. R.; Liu, Y.; Lugovoy, E.; Link, O.; Faubel, M.; Buck, U.; Winter, B.; Abel, B. Nature Chemistry, 2010, 2, 274.

(8) Sagar, D. M.; Bain, C. D.; Verlet, J. R. R. J. Am. Chem. Soc. 2010, 132, 6917.

(9) Wang, C.-R.; Lu, Q.-B. Angew. Chem. Intl. Ed. 2007, 46, 6316.

(10) Verlet, J. R. R.; Bragg, A. E.; Kammrath, A.; Cheshnovsky, O.; Neumark, D. M. Science 2005, 307, 93.

(11) Hammer, N. I.; Shin, J-W.; Headrick, J. M.; Diken, E. G.; Roscioli, J. R.; Weddle, G. H.; Johnson, M. A. Science 2004, 306, 675. 
(12) Nordlund, D.; Ogasawara, H.; Bluhm, H.; Takahashi, O.; Odelius, M.; Nagasono, M.;

Pettersson, L. G. M.; Nilsson, A. Phys. Rev. Lett. 2007, 99, 217406.

(13) Marsalek, O.; Uhlig, F.; VandeVondele, J.; Jungwirth, P. Acc. Chem. Res. 2012, 45, 23.

(14) Herbert, J. M.; Jacobson, L. D. Int. Rev. Phys. Chem. 2011, 30, 1.

(15) Turi, L.; Sheu, W.-S.; Rossky, P. J. Science 2005, 309, 914.

(16) Turi, L.; Rossky, P. J.; Chem. Rev. 2012, 112, 5641.

(17) Schnitker, J.; Motakabbir, K.; Rossky, P. J.; Friesner, R. Phys. Rev. Lett. 1988, 60, 456.

(18) Rossky, P. J.; Schnitker, J. J. Phys. Chem. 1988, 92, 4277.

(19) Barnett, R. N.; Landman, U.; Nitzan, A. J. Chem. Phys. 1988, 89, 2242.

(20) Sprik, M.; Klein, M. L. J. Chem. Phys. 1988, 89, 1592.

(21) Kotler, Z.; Neria, E.; Nitzan, A. Comput. Phys. Commun. 1991, 63, 243.

(22) Webster, F. ; Rossky, P. J.; Friesner, R. A. Comput. Phys. Commun. 1991, 63, 494.

(23) Staib, A.; Borgis, D. J. Chem. Phys. 1995, 103, 2642.

(24) Boero, M.; Parrinello, M.; Terakura, K.; Ikeshoji, T.; Liew, C. C. Phys. Rev. Lett. 2003, $90,226403$.

(25) Herbert, J. M.; Head-Gordon, M. Proc. Natl. Acad. Sci. U.S.A. 2006, 103, 14282.

(26) Frigato, T.; VandeVondele, J.; Schmidt, B.; Schütte, C.; Jungwirth, P. J. Phys. Chem. A 2008, 112,6125 .

(27) Marsalek, O.; Uhlig, F.; Frigato, T.; Schmidt, B.; Jungwirth, P. Phys. Rev. Lett. 2010, $105,043002$.

(28) Uhlig, F.; Marsalek, O.; Jungwirth, P. J. Phys. Chem. Lett. 2013, 4, 338.

(29) Barnett, R. N.; Giniger, R.; Cheshnovsky, O.; Landman, U. J. Phys. Chem. A. 2011, 115, 7378.

(30) Ogg, R. A. J. Am. Chem. Soc. 1946, 68, 155. Ogg, R. A. Phys. Rev. 1946, 69, 668. 
(31) Tuttle, Jr., T. R.; Golden, S. J. Phys. Chem. 1991, 95, 5725.

(32) Hameka, H. F.; Robinson, G. W.; Marsden, C. J. J. Phys. Chem. 1987, 91, 3150.

(33) Sobolewski, A. L.; Domcke, W. Phys. Chem. Chem. Phys. 2007, 9, 3818.

(34) Muguet, F. F. Investigations of Diffuse Intermolecular Electronic Systems, Ph.D. Thesis, Texas Tech University, 1992.

(35) Kevan, L. Radiat. Phys. Chem. 1981, 17, 413.

(36) Schnitker, J.; Rossky, P. J. J. Chem. Phys. 1987, 86, 3471.

(37) Wallqvist, A.; Thirumalai, D.; Berne, B. J. J. Chem. Phys. 1987, 86, 6404.

(38) Jonah, C. D.; Romero, C.; Rahman, A. Chem. Phys. Lett. 1986, 123, 209.

(39) Schnitker, J.; Rossky, P. J. J. Chem. Phys. 1987, 86, 3462.

(40) Barnett, R. N.; Landman, U.; Cleveland, C. L.; Jortner, J. J. Chem. Phys. 1988, 88, 4421.

(41) Turi, L.; Borgis, D. J. Chem. Phys. 2002, 117, 6186.

(42) Jacobson, L. D.; Herbert, J. M. J. Chem. Phys. 2010, 133, 154506.

(43) Larsen, R. E.; Glover, W. J.; Schwartz, B. J. Science, 2010, 329, 65.

(44) Casey, J. R.; Kahros, A.; Schwartz, B. J. J. Phys. Chem. B 2013, 117, 14173.

(45) Turi, L.; Madarász, Á. Science, 2011, 331, 1387-c.

(46) Jacobson, L. D.; Herbert, J. M. Science, 2011, 331, 1387-d.

(47) Herbert, J. M.; Jacobson, L. D. J. Phys. Chem. A 2011, 115, 14470.

(48) Bartels, D. M. J. Chem. Phys. 2001, 115, 4404.

(49) Coe, J. V.; Williams, S. M.; Bowen, K. H. Int. Rev. Phys. Chem. 2008, 27, 27.

(50) Griffin, G. B.; Young, R. M.; Ehrler, O. T.; Neumark, D. M. J. Chem. Phys. 2009, 131, 194302.

(51) Barnett, R. N.; Landman, U.; Cleveland, C. L.; Jortner, J. Phys. Rev. Lett. 1987, 59, 881.

(52) Barnett, R. N.; Landman, U.; Cleveland, C. L.; Jortner, J. J. Chem. Phys. 1988, 88, 4429. 
(53) Madarász, Á.; Rossky, P. J.; Turi, L. J. Chem. Phys. 2009, 130, 124319.

(54) Turi, L.; Madarász, Á.; Rossky, P. J. J. Chem. Phys. 2006, 125, 014308.

(55) Jacobson, L. D.; Herbert, J. M. J. Am. Chem. Soc. 2011, 133, 19889.

(56) Berendsen, H. J. C.; Postma, J. P. M.; van Gunsteren, W. F.; Hermans, J. Intermolecular Forces (Ed. Pullman, B.) Reidel, Dordrecht, 1981, pp. 331-342. Toukan, K.; Rahman, A. Phys. Rev. B 1985, 31, 2643.

(57) Turi, L. J. Chem. Phys. 2014, 140, 204317.

(58) Voora, V. K.; Ding, J.; Sommerfeld, T.; Jordan, K. D. J. Phys. Chem. B 2013, 117, 4365.

(59) Morrison, M. A.; Collins, L. A. Phys. Rev. A 1978, 17, 918.

(60) Phillips, J. C.; Kleinman, L. Phys. Rev. 1959, 116, 287.

(61) Cohen, M. H.; Heine, V. Phys. Rev. 1961 122, 1821.

(62) Smallwood, C. J.; Larsen, R. E.; Glover, W. J.; Schwartz, B. J. J. Chem. Phys. 2006, 125, 074102.

(63) Turi, L.; Gaigeot, M.-P.; Levy, N.; Borgis, D. J. Chem. Phys. 2001, 114, 7805.

(64) Allen, M. P.; Tildesley, D. J. Computer Simulation of Liquids Clarendon, Oxford, 1987.

(65) Madarász, Á.; Rossky, P. J.; Turi, L. J. Phys. Chem. A 2010, 114, 2331.

(66) Marsalek, O.; Uhlig, F.; Jungwirth, P. J. Phys. Chem. C 2010, 114, 20489.

(67) We note that classification of excess electronic states (i.e. interior state vs. different surface states) based on the radii of gyration necessarily contains certain degree of arbitrariness. For example, the radius of gyration of a uniform sphere is $\sim 77 \%$ of the physical radius of the sphere.

(68) Makov, G.; Nitzan, A. J. Phys. Chem. 1994, 98, 3459.

(69) Tang, Y.; Shen, H.; Sekiguchi, K.; Kurahashi, N.; Mizuno, T,; Suzuki, Y.-I.; Suzuki, T. Phys. Chem. Chem. Phys. 2010, 12, 3653. 
(70) Shreve, A. T.; Yen, T. A.; Neumark, D. M. Chem. Phys. Lett., 2010, 493, 216.

(71) We note that this observation also holds for even smaller clusters ( $n=20$ and 30$)$, as seen from test simulations.

(72) Ayotte, P.; Johnson, M. A. J. Chem. Phys. 1997, 106, 811.

(73) Jou, F.-Y.; Freeman, G. R. J. Phys. Chem. 1977, 81, 909. Jou, F.-Y.; Freeman, G. R.

Can. J. Chem. 1979, 57, 591. 